\title{
Género y trabajo: hacia una agenda nacional de equilibrio trabajo-familia en Chile
}

\author{
Gender and Labor: Toward a National Agenda on \\ Labor-Family Balance in Chile
}

\author{
Verónica Gómez-Urrutia (D) http://orcid.org/0000-0002-2399-7566 \\ Universidad Autónoma de Chile,Chile,gomezver@gmail.com
}

Andrés Jiménez-Figueroa (D) http://orcid.org/0000-0001-8324-0250

Recepción:

04/07/2018

Aprobación:

$11 / 10 / 2018$

\author{
Universidad de Talca, Chile, anjimenez@utalca.cl
}

\begin{abstract}
This paper seeks to explore discourses on the possibilities and limitations identified by relevant actors in the sphere of paid work to set up a national agenda on labor-family policies that are compatible with gender equality in Chile. The study is based on in-depth interviews with managers of organizations in different productive sectors, workers' representatives, legislators and officers of the Chilean government. The interviews sought to establish their perceptions on the importance of labor-family balance and policies aimed to promote it, the place of such policies in the political agenda and the conditions necessary to successfully implement them. Two main obstacles to set a national gender-friendly agenda on work-family policies were identified: first, the persistent identification of carework as a female responsibility, thereby, a situation that only affects female workers. Second, the discursive tension between the ideas of productivity and worker's welfare.
\end{abstract}

Key words: paid work, carework, gender, public policy, discursive institutionalism.

Resumen: El presente trabajo busca reconstruir los discursos sobre las posibilidades y limitaciones que surgen desde el mundo del trabajo remunerado para hacer posible instalar una agenda de conciliación de responsabilidades laborales y familiares, con equidad de género, en Chile. El estudio se basa en entrevistas en profundidad a directivos de organizaciones de varios sectores productivos, representantes de trabajadores/as, del Ejecutivo y legisladores/as sobre medidas de conciliación trabajo-familia, su importancia en la agenda laboral y las condiciones necesarias para su implementación. Entre los resultados se encontró que la persistente identificación de las responsabilidades familiares como un ámbito femenino, y la tensión existente entre las ideas de productividad -asociada con largas jornadas de trabajo- y bienestar de los/as trabajadores constituyen dos obstáculos claros para relacionar los discursos, hoy divergentes, sobre trabajo, familia y equidad de género en Chile.

Palabras clave: trabajo remunerado, cuidado, género, políticas públicas, institucionalismo discursivo. 


\section{Introducción}

La distribución del trabajo remunerado frente al no remunerado y sus implicaciones de género han adquirido creciente importancia en la agenda de desarrollo social internacional ${ }^{1}$ : cambios en las formas de constitución de la familia, envejecimiento demográfico, la entrada masiva de las mujeres al mercado de trabajo y la caída sostenida en la tasa de fertilidad han levantado numerosas interrogantes sobre la capacidad futura de las sociedades para conciliar los ámbitos productivo y reproductivo de una manera que promueva la equidad de género.

En cuanto arreglos institucionales, los modos de distribución del trabajo socialmente necesario suponen una asignación de valor -tanto simbólico como material- para cada tipo de trabajo, a menudo expresado como recompensas monetarias y como derechos sociales asociados al mismo. Por ello, también juegan un rol central desde la perspectiva de la justicia distributiva y del reconocimiento de la contribución de los individuos a la sociedad (Fraser, 1997), así como la distribución de oportunidades de vida para hombres, mujeres y niños.

Históricamente, la división sexual del trabajo -hombres proveedores, mujeres cuidadoras- supuso una especialización a lo largo de líneas de género que relegó el trabajo reproductivo en el contexto de la familia -las tareas domésticas y de cuidado- al espacio de lo privado, invisibilizándolo como trabajo socialmente valioso y configurando un tipo de desigualdad enraizado en el propio modo de organización social. La demanda por mayor igualdad de género ha puesto de relieve la importancia del trabajo doméstico y de cuidado como un elemento clave para el bienestar de los individuos y las sociedades, relevando al mismo tiempo las tensiones implícitas en el proyecto de conciliar las demandas de este tipo de labor con las que impone el trabajo remunerado. Dicha tensión sigue estando hoy en el centro de las desigualdades de género en términos de las oportunidades para desarrollar talentos, generar un ingreso propio y decidir sobre él (Adatti et al., 2018). La distinción también cruza las posibilidades de vincular el trabajo no solo con la productividad económica, sino también con el bienestar y el desarrollo armónico de las capacidades humanas.

1 Este artículo fue elaborado en el marco del proyecto Fondecyt 1150250 “Trabajo y familia: hacia un modelo para el establecimiento de una agenda nacional de equilibrio y co-responsabilidad en materia laboral y familiar en Chile", financiado por la Comisión Nacional de Investigación Científica y Tecnológica (Conicyt) de Chile. 
En ese contexto, esta investigación busca reconstruir los discursos sobre las condiciones, posibilidades y limitaciones que surgen desde el mundo laboral remunerado para hacer posible instalar una agenda de conciliación de responsabilidades laborales y familiares, con equidad de género, en Chile. Se buscó identificar los puntos de convergencia y divergencia que diversos actores relevantes en el mundo del trabajo remunerado -empleadores, sindicatos y representantes del Estado- plantean como clave para avanzar en una agenda de equilibrio trabajo-familia en Chile, y si esta es, finalmente, una prioridad política o no.

Metodológicamente, el estudio se basa en el levantamiento de información primaria sobre las percepciones de directivos de gestión de personas, representantes de los/as trabajadores/as, del Ejecutivo y legisladores/as sobre medidas de conciliación trabajo-familia, su importancia en la agenda laboral y las condiciones necesarias para su implementación, de manera que propendan a una mayor igualdad de género. Con ese propósito, el artículo está organizado en cuatro apartados: en el primero, se propone un marco analítico para situar la cuestión de las agendas gubernamentales y los llamados pactos discursivos en legislación y política pública; en el segundo, se detalla la metodología del estudio; en el tercero, se presentan los principales resultados del análisis de datos; y el cuarto y último contiene las conclusiones de la investigación.

\section{Trabajo, familia y género}

En Chile, como ocurre en casi todas las sociedades, la distribución de las formas de trabajo socialmente relevantes está marcada por el género. Los hombres tienen tasas de participación laboral proporcionalmente mayores que las mujeres en todos los niveles educativos; mientras, la participación femenina está claramente condicionada por el nivel de educación formal, la edad y el número de hijos/as, como factores clave. Para las mujeres que logran insertarse en el mercado laboral, ello supone con frecuencia una tarea doble, ya que siguen siendo responsables principales (si no únicas) de las tareas del hogar. Según el think thank Comunidad Mujer (2017), la carga global de trabajo en una pareja chilena -que representa la suma simple entre labores remuneradas y no remuneradas- llega a 11,6 horas diarias para las mujeres y a 9,7 horas para los hombres.

Las encuestas de uso del tiempo aplicadas por el Instituto Nacional de Estadísticas (INE, 2017) corroboran este dato: en un día laboral típico, las mujeres destinan en promedio, a nivel nacional, 5,89 horas al trabajo no 
remunerado, y los hombres, 2,74 horas. Esta brecha aumenta los fines de semana, cuando las mujeres reportan dedicar 6,12 horas, y los hombres 3,5, en media, al trabajo no remunerado, lo cual tiene impactos directos en los tiempos que pueden dedicarse al trabajo remunerado o al ocio y descanso.

Esta distribución de responsabilidades también impacta en las posibilidades que hombres y mujeres tienen para generar un ingreso propio. Las brechas salariales (por sexo), que en Chile pueden llegar al 30\% (INE, 2017), han sido atribuidas, al menos parcialmente, al "impuesto maternidad": aquella diferencia que es solo explicable por el hecho de ser mujer con hijos/as (Budig et al., 2012; Oesch et al., 2017). Asimismo, según la Encuesta de Caracterización Socioeconómica Nacional (CASEN) de 2015, de la población que se encuentra fuera del mercado laboral por razones de trabajo doméstico o de cuidado, ${ }^{2} 20,9 \%$ son mujeres y $0,4 \%$ son hombres (Ministerio de Desarrollo Social, 2017a).

Esta disparidad se acentúa por clase social: entre las mujeres del $20 \%$ de menores ingresos, un 30\% declara no buscar empleo fuera del hogar por razones familiares, frente a un 10\% que expone la misma razón en el 20\% de mayores ingresos de la población (Ministerio de Desarrollo Social, 2017a). Quienes tienen menores ingresos encuentran más desincentivos para ocuparse de manera remunerada, ya sea porque enfrentan la perspectiva de empleos de bajo ingreso y pocas oportunidades de promoción, o bien porque tercerizar el cuidado resulta poco viable por motivos económicos o culturales, cuestión que sí pueden hacer quienes pueden pagar un tercero (por lo general otra mujer) para hacerse cargo de dichas tareas. Ello explica que el $76 \%$ de los mayores de 15 años que no tienen ingresos propios en Chile sean mujeres (Ministerio de Desarrollo Social, 2017b).

La idea, culturalmente arraigada, según la cual la familia es una responsabilidad sobre todo femenina ha sido reforzada hasta tiempos recientes por la política pública. La legislación en esta área se ha orientado a proteger la maternidad en el contexto de los derechos laborales, como la prohibición de condicionar contrataciones a la no existencia de embarazo, el fuero maternal o los derechos de amamantamiento.

Solo recientemente se han aprobado medidas que buscan promover la incorporación activa de los varones a la esfera del cuidado familiar, como la Ley núm. 20.545 (2011), que prolonga el descanso posnatal para mujeres

2 Corresponde a las personas que no buscaron trabajo en las cuatro semanas anteriores a la encuesta por alguna de las siguientes razones: a) no tiene con quién dejar a los niños; b) no tiene con quién dejar a adultos mayores; c) no tiene con quién dejar a otro familiar; o d) quehaceres del hogar. 
trabajadoras a seis meses, con la posibilidad de traspasar al padre parte del tiempo de descanso; la Ley núm. 20.761 de 2014, que extiende a los padres el derecho de alimentación a sus hijos/as, y el Seguro para el Acompañamiento de Niños y Niñas, conocida como Ley SANNA, vigente a partir de 2018. ${ }^{3}$ Todas estas medidas procuran proteger espacios para que las mujeres -y en mucho menor medida, los hombres- puedan cuidar hijos/as pequeños/as sin posponer o sacrificar sus posibilidades laborales.

La legislación nada dice de otras responsabilidades, como niños/as en edad escolar (a menos que estén afectados por una discapacidad o enfermedad grave) o adultos mayores. Otras medidas posibles, como la flexibilización de la jornada de trabajo (horarios de entrada y salida diferidos, por ejemplo), la existencia de permisos extraordinarios -que deban compensarse o no- para atender situaciones familiares puntuales, bancos de horas u otras quedan sujetas a la negociación posible entre las organizaciones y sus colaboradores/as.

Existen algunos intentos por normar la conciliación trabajo-familia a nivel de políticas no vinculantes. En 2006, el entonces Servicio Nacional de la Mujer (Sernam $)^{4}$ lanzó un Código de buenas prácticas laborales y no discriminación, que propone medidas para mejorar la conciliación trabajo-familia, facilitando el acceso de las mujeres a la capacitación y el desarrollo profesional. La adhesión a las normas del código da a las organizaciones públicas y privadas la opción de obtener una certificación (el "Sello Iguala") en la norma chilena NCh 3262. Sobre la base de este código, la Confederación de la Producción y del Comercio (CPC) adoptó ese mismo año una "Guía de buenas prácticas laborales sobre no discriminación en la empresa", la cual incluye una sección sobre "Conciliación de responsabilidades laborales con obligaciones familiares", donde se insta a las organizaciones a impulsar una cultura organizacional amigable con las responsabilidades laborales y parentales de hombres y mujeres, en la medida que ello sea compatible con las necesidades de la empresa (Faúndez et al., 2013). Todas estas medidas son voluntarias y, por tanto, dependen de la decisión de la empresa.

3 Esta última consiste en un seguro obligatorio para que los padres y madres trabajadores de niños y niñas afectados por una condición grave de salud puedan ausentarse de su trabajo para proporcionar cuidados durante un tiempo determinado, recibiendo durante ese periodo un subsidio que reemplace total o parcialmente su remuneración o renta mensual.

4 En 2017, el Sernam pasó a llamarse Servicio Nacional de la Mujer y Equidad de Género, Sernameg, al crearse el Ministerio de la Mujer y Equidad de Género. 
En 2006, la entonces presidenta Bachelet también dictó un Instructivo Presidencial sobre Buenas Prácticas Laborales para la administración del Estado, que plantea la necesidad de incorporar medidas para compatibilizar la vida laboral y familiar en la gestión de personas de cada servicio. En septiembre de 2013 comenzó a regir en Chile el Protocolo de Vigilancia de Riesgos Psicosociales en el trabajo, conocido como ISTAS 21, que incluye la dimensión doble presencia (trabajo-hogar) como un riesgo psicosocial, siendo esta la única dimensión donde no se exigen acciones de intervención si muestra indicadores negativos o riesgosos para los/as trabajadores (Ministerio de Salud de Chile, 2017). Todas estas medidas presentan la característica de seguir orientadas principalmente hacia las mujeres, con los varones en un rol subsidiario; o bien de no tener carácter vinculante que asegure su aplicación de manera generalizada, limitando su impacto.

\section{Pactos discursivos y la conciliación trabajo-familia}

En este contexto, cabe preguntarse por las posibilidades de impulsar, desde la política pública, un conjunto de medidas que promuevan la conciliación trabajo-familia con equidad de género. El presente análisis considera las políticas de conciliación trabajo-familia como aquellas que hacen posible compatibilizar las responsabilidades familiares (trabajo doméstico y de cuidado, sobre todo) con las laborales, y pueden ser implementadas tanto por el Estado como por las organizaciones que proveen empleo remunerado (Greenhaus et al., 2003).5 Así van más allá de la legislación laboral. Aquí se presenta una primera distinción importante: si bien estas políticas son distinguibles de la legislación laboral, están claramente condicionadas por ella. Por ejemplo, la extensa jornada laboral chilena ( 45 horas por semana) determina el tiempo no laboral disponible. Las formas en que dicha jornada puede distribuirse o fraccionarse están establecidas por ley, lo cual puede facilitar u obstaculizar la conciliación.

Una segunda distinción relevante es que las políticas de conciliación trabajo-familia no son neutras en términos de género: pueden reforzar roles tradicionales o ayudar a desmantelarlos. Por ello, para que propendan a una mayor igualdad de género, estas políticas deben promover la corresponsabilidad; esto es, la redistribución equitativa de las responsabilidades familiares

5 En otros contextos, como el anglosajón, se prefiere el concepto equilibrio trabajo-vida (work-life balance) (Casper et al., 2018), en el entendido de que los aspectos no laborales comprenden no solo a la familia, sino también a responsabilidades comunitarias y el derecho al ocio. En este artículo se utilizará el concepto más restringido. 
entre hombres y mujeres, pero también entre las familias y el Estado (OIT y PNUD, 2009; OIT, 2013). Aquí la legislación laboral también provee un marco de referencia que condiciona las políticas: por ejemplo, asegurar servicios de cuidado infantil o de adultos mayores para los/as trabajadores/as favorece la conciliación; no obstante, si estos servicios están destinados solo a las mujeres, no favorecerán la corresponsabilidad. Un ejemplo de ello es la expansión de las salas-cuna públicas en Chile a partir de 2006: si bien favorece la conciliación, el servicio estuvo orientado a las madres, en concordancia con el artículo 203 del Código del Trabajo chileno, en el cual el derecho a la sala-cuna es exclusivo de la trabajadora. ${ }^{6}$ Así se reforzó la idea de que cuidar niños/as es una responsabilidad femenina.

Desde esta perspectiva, la cuestión que aquí se aborda muestra al menos dos condicionantes ideológicos importantes: por una parte, los límites entre el tiempo laboral y el familiar; por otra, la división sexual del trabajo, que supone la preeminencia de los hombres en el mundo "productivo" (el trabajo remunerado) y la de las mujeres en el "reproductivo", la familia. Estas condicionantes son relevantes porque proporcionan un entorno ideológicodiscursivo favorable o desfavorable a ciertos temas como asuntos de política pública, condicionando la manera en que se los define y negocia (Anderson, 2003; Sabatier, 2007; Kingdon, 2003). Tanto la definición del problema como las posibilidades de incluirlo en la agenda pública dependen de los recursos organizacionales, discursivos y de poder con que cuentan los actores, así como de las oportunidades políticas existentes (Fischer y Gottweis, 2012; Schmidt, 2014). Por ello, el entorno ideacional donde se mueven las políticas públicas sobre trabajo y familia resulta clave para identificar las posibilidades de instalar una agenda sobre el tema que lo reconceptualice como un problema de interés público, y no privado, el cual involucra a trabajadores/as, empleadores y el Estado.

El marco de análisis de este trabajo se adhiere a los supuestos de lo que Schmidt (2014) ha denominado institucionalismo discursivo: una mirada que considera que las instituciones son también (o portan) discursos que pueden ser ideológicos, pragmáticos u oportunistas, pero que -más allá de su contenido sustantivo- son movilizadas en un contexto sociocultural específico y con recursos determinados. Así, ciertas ideas pueden ser más afines a determinados contextos, lo cual influye en su instalación como parte del debate público. Ello condiciona la posibilidad de establecer un pacto social que es primero, siempre, un pacto discursivo: los actores relevantes

6 La política de salas-cuna incluyó también a mujeres estudiantes o que estuvieran buscando trabajo, pero no a los padres, manteniendo el sesgo de género. 
acuerdan puntos convergentes, lo cual puede suponer la resignificación de algunos términos o categorías en el proceso de interpretación del problema. Eso no significa relegar a un segundo plano la cuestión del poder, pues, como apunta Schmidt (2014: 7), el discurso y las acciones de los agentes constituyen también una respuesta a intereses y realidades materiales.

Desde esta mirada, la elaboración e implementación de las políticas sobre conciliación trabajo-familia pueden ser entendidas como situadas en un campo discursivo donde se construyen y reconstruyen las legitimidades sociales, tanto en términos de los límites de la acción del Estado, como a lo que se define como propio en términos de género. Esta cuestión implica negociar -en términos de sentido- la distinción entre lo público (el trabajo) y lo privado (la familia), y, dentro de esta última, las posibilidades que hombres y mujeres tienen para negociar roles en relación con el cuidado, el trabajo doméstico y la provisión de ingreso. Las cuestiones referidas a género y familia tienen, además, una dimensión valórica (la importancia social otorgada al rol materno, por ejemplo), lo cual implica que se requiere de un acuerdo político, relativamente fuerte y, por ello, un mayor grado de articulación discursiva, para lograr institucionalizar una política pública en esta área (Gómez, 2014). Asimismo, Blofield y Martínez (2014) y Staab (2017) destacan cómo la alteración del orden de género ahora vigente exigiría revisar una parte importante de la arquitectura de las leyes laborales y de bienestar social, que históricamente han asumido el trabajo femenino no remunerado -ya sea como trabajo doméstico y de cuidado o como actividades comunitarias no pagas- como base de las políticas, en especial las sociales, como parte del rol ciudadano de las mujeres. Por ello, la promoción de políticas de conciliación trabajo-familia con corresponsabilidad supone redefinir algunos términos básicos de este campo discursivo y su aceptación como parte del actuar legítimo del Estado proveniente de los actores relevantes del mundo laboral: el propio Estado, las organizaciones productivas y de servicios, y sus trabajadores/as.

\section{Metodología}

En términos de los participantes en este estudio, se identificaron tres grupos de actores relevantes para la discusión sobre políticas de conciliación trabajo-familia, utilizando el criterio de selección de informantes estratificado intencional, que busca identificar a participantes de diferentes estratos de un mismo colectivo social (Izcara, 2014) y que se resumen en el Cuadro 1.7

7 Los cuadros se encuentran en el Anexo, al final del presente artículo (Nota del editor). 
Para los dos primeros grupos, se contactó a organizaciones que tuvieran al menos 100 trabajadores/as, de distintos sectores productivos: manufacturas, servicios y sector público. Este criterio obedece al hecho de que el sector productivo al cual pertenece la organización plantea desafíos específicos para la organización de los tiempos de trabajo, o bien la característica de contar con una fuerza de trabajo predominantemente masculina o femenina. Al final, 11 organizaciones aceptaron participar en el estudio. De estas, tres pertenecen al sector público, cuatro al sector servicios (comercio y servicios bancarios) y cuatro al sector manufacturero. Para el caso de los/as parlamentarios, se contactó a todos los/as miembros de las Comisiones de Trabajo y Previsión Social, tanto en la Cámara como en el Senado. Cuatro de ellos/as aceptaron ser entrevistados personalmente, dos hombres y dos mujeres; en términos de filiación partidaria, dos militaban en partidos de centro-izquierda, uno de centro-derecha y uno se declaró independiente. En los Ministerios, se procuró a informantes que tuvieran directa relación con temas que cruzaran la conciliación trabajo-familia. En total, se realizaron 52 entrevistas semiestructuradas, entre 2015 y 2017, al mismo número de participantes, con una duración promedio de 60 minutos cada una.

En todos los casos, se firmó una carta compromiso en la cual tanto las organizaciones participantes como los/as investigadores se comprometieron a respetar la voluntariedad, confidencialidad y el anonimato de quienes aceptaran actuar como informantes. Además, cada informante recibió y firmó un formulario de consentimiento informado individual. La técnica de recolección de información fue la entrevista semiestructurada, con pautas homólogas, esto es, que cubrían los mismos temas para cada tipo de participante. Se escogió la entrevista semiestructurada por ser un instrumento que permite al investigador/a acceder a la perspectiva del sujeto estudiado: comprender sus categorías mentales, sus interpretaciones, sus percepciones y sus sentimientos, los motivos de sus actos sobre la base de un cuestionario con una secuencia lógica, pero que es no estandarizado y flexible (Izcara, 2014; Wengraf, 2004).

El análisis del corpus representado por las entrevistas privilegió identificar y comparar tres ejes discursivos, definidos grosso modo, como: a) la necesidad percibida de implementar reformas laborales que faciliten la conciliación familia-trabajo, y las razones que las justifican; b) la necesidad de que estas políticas fomenten la corresponsabilidad, en los términos definidos con anterioridad en este texto; y c) las oportunidades/barreras percibidas para implementar políticas que faciliten la conciliación familiatrabajo. Para ello, se utilizó un proceso de categorización abierta primero 
y estructural después (MacQueen et al., 2008; Saldaña, 2009), ocupando el software Atlas.ti, versión 7. Esto es, primero se hizo una codificación que identificara los temas o categorías más recurrentes en los discursos, y su posible asociación con otras ideas. Luego, se usó la codificación estructural, que aplica ideas o conceptos clave para la investigación de cada segmento del texto (Saldaña, 2009), definiendo tres grandes unidades conceptuales clave o macrocategorías resumidas en el Cuadro 2, que, a su vez, daban origen a dimensiones conceptuales asociadas. El análisis consideró categorías y dimensiones emergentes, surgidas del propio discurso de los/as participantes.

Posteriormente, estas categorías fueron ordenadas en términos de las relaciones lógicas establecidas entre ellas - esto es, la estructura base del discurso- y se incluyeron las valoraciones que los/as informantes hicieron de dichas categorías. Al final, las estructuras encontradas para cada uno de los tres grupos fueron comparadas sistemáticamente, con énfasis en establecer los puntos de convergencia y divergencia de los discursos, en el entendido de que estos no son monolíticos y presentan contradicciones internas. Dicho proceso fue revisado por una profesional externa a la investigación, quien actuó como consultora para evitar sesgos derivados de nuestro propio interés investigativo.

\section{Resultados}

Para efectos de claridad en la exposición, se ordenarán los hallazgos en función de los ejes discursivos utilizados para el análisis. En términos de la necesidad percibida de implementar medidas que faciliten la conciliación familia-trabajo, una primera constatación es que pocas empresas - de las que participaron en este estudio, pero también a nivel nacional (Bosch, et al. 2014) - tienen políticas de conciliación trabajo-familia, centrándose en el cumplimiento de la ley laboral vigente. Las razones planteadas para ello se fundamentan, básicamente, en que si bien se atribuye gran importancia a la familia y al adecuado cumplimiento de los roles familiares para el bienestar de los/as trabajadores/as, este es un ámbito que escapa a la responsabilidad de las organizaciones, pues se trata de decisiones y modos de organización privados que requieren, por tanto, de soluciones privadas. Así, aunque se reconoce la relevancia de considerar a los trabajadores/as desde el punto de vista de sus realidades familiares, no se considera la dimensión familiar como un factor importante al momento de organizar el trabajo. Los siguientes extractos ilustran el contraste entre estos dos ámbitos: 
El [empleador] no tiene a la familia del trabajador en mente, son cosas bien distintas. Entonces si tú eres soltera, no tienes hijos ni padres [adultos mayores que cuidar], es como la situación ideal. Pero esa gente [sin responsabilidades familiares] es poca. Aquí se castiga a quienes tenemos familia, porque es [...] como que no fuera parte de lo que la gente [trabajadores/as] necesita (dirigente sindical, mujer, sector servicios). ${ }^{8}$

Sí, claro, la familia [de los trabajadores/as] es muy importante, la idea de que son personas. No números. Eso está contemplado [en la gestión de personas]. Pero no, no hay una política formal [de conciliación trabajo-familia]. Yo diría que más bien es un tema, no sé, como de sentido común, de respeto al reconocer que hay espacios personales importantes [...] Pero no me queda la sensación de que sea una cosa que tú digas, o la empresa [diga]: "es parte del trabajo" (directivo de gestión de personas, mujer, sector manufacturero). ${ }^{9}$

Esta lógica interpretativa se refleja en el estatus de las medidas de conciliación trabajo-familia dentro de las organizaciones, que típicamente se encuentran asociadas a la idea de prestaciones en el área de bienestar -en el mismo espacio que las fiestas de Navidad o los convenios con casas comerciales, por ejemplo- y no como parte de la gestión de personas. De las 11 empresas participantes en el estudio, tres declararon tener una política explícita en esta materia. De ellos, en dos casos dicha política estaba altamente formalizada, con reglas elaboradas y un sistema claro para organizar los tiempos familiares. No obstante, los trabajadores/as de estas organizaciones reportaron que la utilización de dichas medidas estaba muy condicionada por las decisiones de supervisores y jefes directos, lo cual en la práctica reintroducía un grado importante de discrecionalidad. Grados de discrecionalidad variable significan reglas ad hoc que pondrían en una situación de menoscabo a quienes sí tienen obligaciones de cuidado por cumplir o están en posiciones de menor poder negociador, como ocurre con las personas con posiciones vistas como "reemplazables" dentro de la organización (Gornick y Meyers, 2009). Esto afecta en particular a las mujeres, sobre todo a las que tienen menores niveles de calificación, pues, debido a los mandatos culturales de género, son quienes habitualmente piden permisos para atender situaciones familiares, las cuales son vistas como interrupciones del desempeño "normal" del trabajador/a. Lo anterior también pone en riesgo sus posibilidades de desarrollo de carrera. Las oportunidades de promoción y ascenso son un ejemplo paradigmático de esta tensión, ya que, por lo general, están vinculadas al cumplimiento de determinadas metas de productividad o entrenamiento que se consideran adecuadas al horario normal

8 Entrevista personal, 18 de enero 2016 (en Santiago).

9 Entrevista personal, 23 diciembre de 2015 (en Talca). 
de trabajo. O bien, las oportunidades de capacitación están relacionadas al compromiso (expresado como permanencia en el lugar de trabajo) que jefes y supervisores perciben en el trabajador/a, y que están condicionadas por la voluntad (léase disponibilidad) del trabajador/a para priorizar los tiempos laborales por sobre los familiares.

La lógica de la conciliación trabajo-familia como un problema privado e individual, y no como uno con raíces estructurales, también permea la discusión sobre la flexibilidad laboral. Según los datos disponibles en Chile, en aquellas empresas que sí tienen medidas de conciliación, en promedio cerca del $70 \%$ de los/as trabajadores/as valora positivamente las medidas de flexibilidad laboral (Fundación Chile Unido, 2017), ${ }^{10}$ tales como permisos con o sin goce de sueldo, horarios de entrada y salida diferidos o semanas comprimidas para tener un día libre o jornada más breve. Cuando estas son parte de una política de gestión de personas y aplicadas con criterios claros pueden, efectivamente, ayudar a organizar los tiempos de manera más armónica; no obstante, en un contexto individualizado y/o donde hay bajo poder de negociación, también representan el riesgo de que la organización colonice espacios familiares que ya estaban garantizados por ley o por negociaciones anteriores si los horarios se organizan en torno a las demandas de la productividad, y no de la vida familiar. Por ello, las organizaciones de trabajadores manifiestan desconfianza frente a posibles medidas de flexibilidad, pues en muchos casos estas aparecen en tensión con derechos laborales ya adquiridos o como parte de una lógica que se define como de precarización laboral y contraria a los intereses de los/as trabajadores/as. ${ }^{11}$

Este debate se vio reflejado a nivel nacional durante la discusión sobre la Reforma Laboral impulsada por la segunda administración de la socialista Michelle Bachelet (2014-2018), que incluyó los llamados "pactos de adaptabilidad": la posibilidad de que trabajadores/as y empresas pudieran establecer algunos acuerdos sobre distribución de jornadas y otros acuerdos de adaptabilidad para trabajadores con responsabilidades familiares, mujeres y jóvenes. Originalmente, esta medida llevaba aparejada una mayor capacidad de negociación de los sindicatos - la titularidad sindical-, que fue vetada durante la administración entrante del centro-derecha Sebastián Piñera (2018-), dejando abiertos espacios de negociación, pero en un contexto de relaciones de poder desiguales, como apunta un miembro del Legislativo:

10 Datos de un estudio en el cual participaron 90 organizaciones nacionales y multinacionales, privadas y públicas, de distintos tamaños y sectores de la economía. El estudio incluye empresas que se presentaron voluntariamente, por lo cual tiene un sesgo de autoselección.

11 Entrevista grupal con 14 dirigentes sindicales, 22 de octubre de 2016 (en Talca). 
Quedamos en el peor de los mundos [después del veto], porque ahora pueden negociarse espacios que antes estaban protegidos, pero sin aumentar el poder de los trabajadores. Entonces es una negociación nominal, porque [el resultado] va a depender de lo que el empleador defina como mejor para los intereses de la empresa. Y aquí [en Chile] hay mucho una cultura empresarial de organizar la vida de las personas en torno a las necesidades del trabajo (senadora, Comisión de Trabajo, partido de centro)..$^{12}$

Una segunda constatación, relacionada con el segundo eje analítico, se refiere a la escasa adhesión a la idea de considerar la corresponsabilidad entre hombres y mujeres para compatibilizar trabajo y familia. El concepto de corresponsabilidad supone que las responsabilidades familiares deben distribuirse entre los miembros adultos de la familia, independiente de su sexo (OIT, 2013). Si bien esta distribución es producto de un acuerdo dentro del grupo familiar, las políticas públicas y las organizaciones pueden ampliar o reducir los márgenes de negociación disponibles para hombres y mujeres, en términos de facilitar o dificultar que las responsabilidades familiares se distribuyan de manera más equitativa.

En términos generales, en las empresas participantes en este estudio se reconoce la demanda por tiempos familiares como no solo privada, sino esencialmente femenina. Esto es entendible desde la perspectiva del referente legal: como ya se indicó, en Chile la mayoría de los derechos relacionados con responsabilidades familiares - como el posnatal y la sala-cuna- están asociados a la trabajadora, reforzando estereotipos culturales. Sin embargo, incluso en organizaciones altamente feminizadas, como el comercio y la banca, la conciliación trabajo-familia aparece como un problema de las trabajadoras, y no del conjunto de la fuerza de trabajo, como muestran los siguientes extractos:

Y te vas a reír, pero los que más piden permiso son los hombres. [En el caso de ellos] es más para cosas personales, el médico, ese tipo de cosas... Pero queda la idea de que son las mujeres [las que piden los permisos], por el tema de los hijos, de la casa. Sí, porque siempre [se dice en las empresas], "no, es que las mujeres viven pidiendo permiso, que no sé qué", no [eso es] mentira, falso (directiva de RR.HH, mujer, sector manufacturero)..$^{13}$

Aquí no tenemos muchos problemas de doble presencia [trabajo-familia] porque la mayoría de nuestros trabajadores son hombres, y son casados. Entonces la cosa de la casa, los hijos, se resuelve de otra manera [sin participación de la empresa] (directivo de RR.HH, hombre, sector manufacturero). ${ }^{14}$

12 Entrevista personal, 02 de marzo 2016 (en Valparaíso).

13 Entrevista personal, 14 de agosto 2015 (en Santiago).

14 Entrevista personal, 28 de julio 2016 (en Talca). 
Esta feminización de las responsabilidades familiares tiene, para las trabajadoras, efectos en dos frentes: por una parte, plantea una tensión entre la satisfacción de necesidades prácticas e inmediatas que hoy afectan más a las mujeres, por la identificación del rol femenino con la familia, y la necesidad estratégica de cuestionar el modelo de trabajador ideal como alguien siempre disponible para los requerimientos de la organización. Hacer esto último implica socavar el estándar de trabajador "ideal" como aquel sin responsabilidades familiares - un estándar frente al cual la mayoría de las trabajadoras aparece como deficiente, problemática-, pero que aún tiene legitimidad en los directivos de las organizaciones y también en los trabajadores sin compromisos familiares. Como señala una de nuestras informantes:

Cuando planteamos la cuestión de los tiempos, de ajustar las metas y objetivos [de desempeño] a la realidad familiar, a mí me dice [el empleador]: "viste, ése es el costo de trabajar con mujeres". Porque la fuerza de venta son, la mayoría, mujeres. " $¡ Y$ cómo tal persona?". Ya, pero ella no tiene hijos y sus padres son todavía jóvenes, pero eso no va a durar toda su carrera, va a tener que escoger (dirigente sindical, mujer, sector bancario). ${ }^{15}$

Este punto tiene implicaciones prácticas concretas en un segundo frente: definir las cuestiones que estarán sobre la mesa de negociaciones entre empleadores y trabajadores como demandas legítimas. Al caracterizarse las políticas de conciliación trabajo-familia como políticas "para mujeres", la demanda se instala en un campo discursivo de legitimidad ambigua, por dos razones principales: una, porque aparece como un interés particular que tiene, por ello, menos relevancia que otras demandas sí percibidas como que afectan al conjunto de la fuerza de trabajo. Una de nuestras informantes lo sintetiza así:

[N]osotros en [el comercio minorista], como la mayoría es mujer, preferimos que se trabaje de lunes a viernes, y el fin de semana no se trabaje. Eso ayuda a la familia. Al [colega] hombre, que es más proveedor, no. Siempre pasa eso [en las negociaciones]: que, por ejemplo, tenemos compañeros que ellos no quieren [dejar de trabajar el fin de semana], a ellos les perjudica, porque tenemos que pensar que el fin de semana es donde vendemos más (dirigente sindical, mujer, sector servicios). ${ }^{16}$

La otra razón es que la literatura sugiere que cuando una demanda se identifica con el cuestionamiento a roles instalados en el imaginario como femeninos, como la maternidad o el cuidado, pierde fuerza (Gómez, 2014; Staab, 2017). En esa lógica, las remuneraciones y beneficios pecuniarios tienen prioridad por sobre otras necesidades, $y$ trabajar en tiempos normalmente

15 Entrevista personal, 17 diciembre 2015 (en Santiago).

16 Entrevista personal, 13 de julio de 2015 (en Talca). 
destinados a la familia - turnos de fines de semana o de noche, feriados y fiestas locales o nacionales- se considera una opción para mejorar el ingreso por quienes no tienen (o delegan en otras personas) responsabilidades familiares o priorizan el rol de proveedor, pues son pagados a una tasa más alta que la jornada habitual. Por ello, suelen ser un punto de negociación contencioso, incluso dentro de las propias organizaciones de trabajadores, que muchas veces no consiguen presentar una demanda común frente al empleador en este punto, o, si lo hacen, esta aparece como secundaria.

$\mathrm{Al}$ mismo tiempo, otra constatación del presente estudio es que con frecuencia se asume que mejores remuneraciones necesariamente solucionarán los temas de conciliación, confundiendo dos problemas que -aunque relacionados- pueden no tener una solución común, como muestra el siguiente extracto:

No es que a la gente no le importe [demandas relacionadas con conciliación trabajofamilia]. Pero uno trabaja por plata y al final las lucas [dinero] son las lucas, especialmente [en un país] con sueldos que son súper bajos. [...] Hay gente que trabaja mucho porque la plata no le alcanza (dirigente sindical, hombre, sector manufacturero). ${ }^{17}$

Esta paradoja se da incluso en empresas altamente feminizadas (como el comercio y la banca), aunque las dirigencias sindicales encabezadas por mujeres otorgan mayor importancia a las demandas por tiempo familiar que sus contrapartes masculinas. Aumentar los sueldos - e igualar las remuneraciones de hombres y mujeres - ciertamente disminuiría la necesidad de trabajar horas extra o en tiempos fuera de la jornada habitual, pero no brinda respuesta a las exigencias familiares cotidianas, que son el mayor obstáculo para la incorporación de las mujeres al mundo laboral remunerado y para sus posibilidades de desarrollo de carrera en una situación de igualdad con los varones.

Desde la perspectiva de las oportunidades/barreras percibidas para implementar políticas que faciliten la conciliación familia-trabajo, una de las categorías más recurrentes fue la productividad laboral. Se trata de una categoría emergente que aparece en una doble vertiente. Por una parte, altos niveles de productividad son vistos como una oportunidad para dar mayor autonomía a los/as trabajadores/as en la organización de los tiempos de trabajo. En contraste, bajos niveles de productividad están asociados discursivamente a la necesidad de extender las jornadas de trabajo y a la reducción de los niveles de autonomía que tienen los niveles operativos de las organizaciones, para definir sus modos de ordenamiento. Los datos disponibles sugieren que aunque la producción por trabajador/a en Chile es relativamente baja cuando se le compara con otros países de la Organización

17 Entrevista personal, 29 diciembre 2015 (en Talca). 
para la Cooperación y el Desarrollo Económicos (OCDE), a la cual Chile pertenece desde 2010, puede considerarse alta en el contexto de otros países latinoamericanos (OCDE, 2018).

Sin embargo, en los discursos tanto de directivos en el área de gestión de personas como de sindicatos y otras asociaciones de trabajadores, la "baja productividad" de la fuerza de trabajo chilena aparece como una categoría recurrente, aunque con orígenes diferentes. Para los empleadores, se trata de una cultura organizacional de trabajo extensivo, más que intensivo; para los/ as trabajadores/as se trata de falta de inversión en capacitación pertinente para desarrollar las tareas de forma más eficiente. Ello ayuda a entender que aunque se mencione con frecuencia la idea de que trabajadores/as con vidas más equilibradas producirán más, esta coexiste con la noción de que alterar los modos de trabajo existentes para facilitar la conciliación redundará en menos horas de trabajo y, por ende, en una productividad todavía más baja. Un miembro del Parlamento sintetiza la cuestión del siguiente modo:

Creo que si el empresario ve que eso [la conciliación trabajo-familia] ayuda a la productividad de su empresa, no me cabe ninguna duda que la van a implementar. La cuestión es quién da el primer paso, quién asume el riesgo y los costos asociados a estas decisiones (diputado, Comisión de Trabajo y Previsión Social, partido de derecha). ${ }^{18}$

La caracterización del cambio requerido para implementar medidas de conciliación como un riesgo ayuda a entender, también, que el bienestar de los/as trabajadores/as se vea como en tensión con el objetivo último de las organizaciones: la sobrevivencia económica. Sin embargo, el presente estudio y otras investigaciones (Bosch et al., 2014; Fundación Chile Unido, 2017) muestran que hay escaso registro acerca del funcionamiento de este tipo de medidas, o de cómo se les podría evaluar más allá de la percepción de los/as trabajadores/as. Incluso entre las organizaciones que cuentan con políticas explícitas de conciliación, los impactos efectivos en la fuerza de trabajo -por ejemplo, en la rotación de personal o en la productividad no son medidos ni registrados sistemáticamente en ninguna de las empresas cubiertas por este estudio, y hay pocas referencias en la literatura para el caso de Chile. La lógica del riesgo, así, no se pone a prueba e ignora que los arreglos actuales ponen el eventual costo de la conciliación en las mujeres, pues se asume que ellas deben cumplir roles productivos sin dejar el reproductivo y asumir la desigualdad de oportunidades que ello genera como parte de una decisión "privada". Esta es una ambivalencia que también se reconoce desde el discurso estatal sobre el empleo femenino, como lo resume uno de nuestros informantes en el poder Ejecutivo:

18 Entrevista personal, 03 de julio 2015 (en Santiago). 
Cuando [el país] se da cuenta que necesita a las mujeres porque la economía está creciendo y porque necesitas llenar los puestos [de trabajo], no necesariamente se le da toda la vuelta a lo que eso implica. Porque adecuar políticas de manejo de recursos humanos para realmente hacerte corresponsable requiere una reflexión bastante más profunda que hacer más salas-cuna. Y porque hay muchas políticas sociales, como la educación preescolar, que todavía están basadas en el trabajo [no remunerado] de las mujeres. Y ahí los temas de mujer importan poco y son un foco de conflicto, porque cuando se quiere criticar es fácil decir: "Ah, esto es antiempleo femenino, se va a encarecer el empleo, va a caer la productividad" (asesora del Ministerio de Trabajo y Previsión Social y del Ministerio de Hacienda). ${ }^{19}$

En este contexto, la discusión sobre la productividad se produce en un marco discursivo que no considera la modificación sustantiva de la división sexual del trabajo, ni los modos actuales de organizar el trabajo productivo, para contemplar la inclusión de las responsabilidades familiares. Estas últimas se ven como resultado de la decisión (personal) de priorizar una esfera por sobre otra y, por ello, como un riesgo para la productividad, especialmente en un entorno que se describe como cambiante y competitivo. El discurso estatal en este punto es ambiguo: si bien se declara la necesidad de avanzar hacia una mayor igualdad de género e incorporar los talentos femeninos al empleo, al mismo tiempo se sostiene la idea de que la mayor contribución social de las mujeres está en el dominio del trabajo reproductivo (Gómez, 2014). Hay allí una omisión respecto de cómo democratizar los roles y tareas de cuidado al interior de la familia, aunque este sea declarado como un objetivo deseable (Ansoleaga y Godoy, 2013).

\section{Discusión y conclusiones}

El análisis presentado en las páginas precedentes sugiere que, a pesar de la creciente incorporación de las mujeres a la fuerza laboral, subsisten barreras importantes para una discusión amplia de los modos de organizar el trabajo productivo y el reproductivo fuera de los moldes tradicionales, que suponen la especialización de tareas por género, y también, una distribución de recompensas desigual para cada tipo de trabajo. Lo anterior ha redundado en una desventaja sistemática de las mujeres en el ámbito económico y en las oportunidades para el desarrollo de talentos por sexo, así como en una sobrecarga significativa por la doble jornada de trabajo que enfrentan quienes cotidianamente deben articular sus responsabilidades de cuidado con las del trabajo remunerado.

19 Entrevista personal, 13 de mayo 2016 (en Santiago). 
Una parte relevante de estas barreras son de orden ideológico y se refieren, sobre todo, a la primacía discursiva de la familia como una esfera desvinculada del trabajo productivo y, por ello, a la distribución del trabajo reproductivo que ocurre dentro de ella como obedeciendo a decisiones puramente personales. Así, los condicionantes culturales y estructurales de estas decisiones -las expectativas de género, pero también la organización de los tiempos de trabajo productivo, la lógica de los incentivos para el desarrollo de carrera y las oportunidades abiertas para hombres y mujeres- son sistemáticamente invisibilizados. Desde esta perspectiva, en los discursos los lugares de trabajo se presentan como neutros en términos de género, a menos que existan disposiciones explícitas en sentido opuesto; por ejemplo, aquellas referidas a las mujeres embarazadas o que están amamantando, que se justifican por motivos biológicos. Las diferencias salariales y en el acceso a puestos de responsabilidad entre hombres y mujeres son vistas como el resultado de la disponibilidad (personal) de los individuos para invertir tiempo, energía y talento en el trabajo remunerado. Ello no considera la expectativa, socialmente sancionada, según la cual el trabajo reproductivo - clave para mantener el bienestar de niños/as, hombres y mujeres en el hogar- es realizado casi exclusivamente por las mujeres. Supone, además, que aquellas con responsabilidades familiares serán menos productivas, pues el foco de su energía estará en el hogar. A pesar de que este supuesto no ha sido avalado por los datos empíricos, estaría en el origen del "impuesto maternidad" (Oesch et al., 2017).

Por eso, los discursos sobre conciliación trabajo-familia aún se centran en la necesidad de facilitar que las mujeres puedan compatibilizar lo que se concibe como su rol principal -el cuidado del hogar y de los hijos/as- con el eventual rol de proveedora, y no en la redefinición de los roles tradicionales, pese al aumento progresivo de hogares chilenos que dependen del ingreso de una mujer, estimado en torno al 33\%, y a que muchos de los cuales son monoparentales con jefatura femenina (Ministerio de Desarrollo Social 2017a).

La ambigüedad del discurso del Estado chileno en esta materia resulta clave. Históricamente, las políticas sociales han descansado, de manera importante, sobre el trabajo femenino no remunerado, ya sea en la forma de cuidados o actividad comunitaria - lo cual Staab (2012) identifica como "maternalismo" en política social-. Como se señaló al inicio de este trabajo, gran parte de la legislación laboral chilena vincula los derechos de cuidado a las mujeres (y en específico a las madres), relegando a los varones a un rol subsidiario y poniendo un marco de referencia sesgado ideológicamente frente a las organizaciones productivas. 
Así, por una parte, se insta al mundo del trabajo a incorporar a las mujeres en condiciones de igualdad; pero, por otra, se omite la importante cuestión de los marcos culturales y legales que presuponen el rol reproductivo de las mujeres, los cuales ponen un límite a la constitución de una agenda integrada de género y trabajo desde el Estado. El efecto señero de estas últimas es clave, incluso en aquellas empresas donde implementan medidas más allá de la legislación laboral, pues podría incentivar la corresponsabilidad disminuyendo las probabilidades de que las demandas se feminicen y, por esa vía, pasen a ser secundarias en las agendas de los propios trabajadores organizados.

Desde el punto de vista de las relaciones de poder, quienes podrían impulsar este debate -las organizaciones de trabajadores- presentan escisiones internas sobre esta materia y además cuentan con un poder de negociación limitado, como lo muestra el debate sobre la flexibilidad laboral. En un contexto de bajo poder negociador, es razonable suponer que nuevas formas de organización del trabajo serán miradas con desconfianza por parte de los/as trabajadores/as, ya que históricamente en Chile la flexibilización ha sido entendida como desregulación (Sisto, 2009). Las organizaciones plantean que una forma de sobrevivir a entornos de trabajo cambiantes y globalizados es aumentar el valor agregado (la productividad) o bien, flexibilizar los marcos legales que rigen el trabajo, para permitir respuestas más ágiles frente a cambios en los mercados; pero todavía no es posible dimensionar con plenitud los costos asociados desde un punto de vista económico, social, familiar y personal, y, particularmente, cómo estos costos se distribuirán entre empresas, trabajadores/as y el Estado, además de su impacto en la (des)igualdad de género.

Contar con investigaciones que aporten en esta línea es muy importante en un contexto donde las nuevas formas de flexibilidad o trabajo parcial se abren como una posibilidad de empleo para quienes tienen el mayor peso de las responsabilidades familiares (las mujeres), en el entendido de que a ellas les corresponde desplegar estrategias privadas de conciliación. Flexibilizar sin considerar las relaciones entre trabajo productivo y reproductivo presenta el riesgo real de que, otra vez, los eventuales costos y beneficios de nuevos arreglos institucionales se distribuyan desigualmente a lo largo de los clivajes de género y clase social, perpetuando las inequidades existentes. Por ello, la constitución de una agenda efectiva de conciliación trabajo-familia requiere evidenciar los vínculos entre el trabajo productivo y el reproductivo, así como el papel crucial que este último tiene en la manutención del bienestar social, lo cual es responsabilidad de toda la sociedad y no solo de las mujeres. 
Convergencia Revista de Ciencias Sociales, núm. 79, 2019, Universidad Autónoma del Estado de México

\section{Referencias}

Addati, Laura et al. (2018), "Care Work and Care Jobs for the Future of Decent Work". Disponible en: https://www.ilo.org/wcmsp5/groups/public/---dgreports/---dcomm/--publ/documents/publication/wcms_633135.pdf [22 de septiembre de 2018].

Anderson, James (2003), Public policymaking: An introduction, Estados Unidos: Houghton. Ansoleaga, Elisa y Godoy, Lorena (2013), "La maternidad y el trabajo en Chile: Discursos actuales de actores sociales", en Polis, vol. 12, núm. 35. Disponible en: https://dx.doi. org/10.4067/S0718-65682013000200015 [22 de diciembre de 2016].

Blofield, Merike y Martínez, Juliana (2014), "Maternalism, co-responsibility and social equity. A typology of work-family policies”, en Social Politics, núm. 4, vol. 21. Disponible en: https://doi.org/10.1093/sp/jxu015 [22 de diciembre de 2016].

Bosch, María José et al. (2014), "Estudio percepciones y prácticas de conciliación y corresponsabilidad en organizaciones públicas y privadas”. Disponible en: http:// www.ese.cl/publicaciones/estudio-percepciones-y-practicas-de-conciliacion-ycorresponsabilidad-en-organizaciones-publicas-y-privadas/ [23 de marzo de 2015].

Budig, Michelle J. et al. (2012), "The Motherhood Penalty in Cross-National Perspective: The Importance of Work-Family Policies and Cultural Attitudes”, en Social Politics vol. 19, núm. 2. Disponible en: https://doi.org/10.1093/sp/jxs006 [25 de marzo de 2018].

Casper, Wendy et al. (2018), “The Jingle-Jangle of Work-Nonwork Balance: A comprehensive and meta-analytic review of its meaning and measurement", en Journal of Applied Psychology, vol. 103, núm. 2. Disponible en: http://psycnet.apa.org/doiLanding?doi $=10.1037 \% 2$ Fapl0000259 [25 de marzo de 2018].

Comunidad Mujer (2017), Mujer y trabajo: Uso del tiempo y la urgencia por compartir las tareas domésticas y de cuidado. Disponible en: http://www.comunidadmujer.cl/ biblioteca-publicaciones/wp-content/uploads/2017/04/BOLETIN-38-marzo2017-baja_vf.pdf [23 de marzo de 2015].

Faúndez, Alejandra et al. (2013), "Guía sistema de gestión de igualdad de género y conciliación de la vida laboral, familiar y personal en las organizaciones”. Disponible en: https://www. minmujeryeg.cl/ministerio/estructura/mujer-y-trabajo/buenas-practicas-laborales-ytrabajo-decente-para-la-igualdad-de-genero/ [23 de octubre de 2015].

Fischer, Frank y Gottweis, Herbert [eds.] (2012), The Argumentative Turn Revisited: Public Policy as Communicative Practice, Estados Unidos: Duke University Press.

Fraser, Nancy (1997), Justice interruptus, Inglaterra: Routledge.

Fundación Chile Unido (2017), "Informe General Mejores Empresas para Madres y Padres que Trabajan 2016". Disponible en: www.chileunido.cl/wp-content/ uploads/2017/01/Informe-General-2016-Estudio-Mejores-Empresas.pdf $[20$ de enero de 2018].

Gómez, Verónica (2014), "Negotiating Gender: Family and Legislation in Chile”, en Australian Feminist Studies, vol. 29, núm. 82. Disponible en: https://www.tandfonline. com/doi/abs/10.1080/08164649.2014.976897 [23 de marzo de 2015].

Gornick, Janet y Meyers, Marcia (2009), "Institutions that support gender equality in parenthood and employment”, en Gornick, Janet y Meyers, Marcia [eds.], Gender equality: transforming family divisions of labor, Inglaterra: Verso.

Greenhaus, Jeffrey et al. (2003), "The relation between work- family balance and quality of life”, en Journal of Vocational Behavior, núm. 63, Estados Unidos: Elsevier. 
INE (Instituto Nacional de Estadística de Chile) (2017), Encuesta Nacional sobre Uso del Tiempo. Principales Resultados. Disponible en: http://historico.ine.cl/enut/files/ principales_resultados/documento_resultados_ENUT.pdf [22 de septiembre de 2018].

Izcara, Simón (2014), Manual de Investigación Cualitativa, México: Fontamara.

Kingdon, John (2003), Agendas, alternatives, and publicpolicies, Estados Unidos: AddisonWesley.

MacQueen, Kathleen et al. (2008), “Team-based codebook development: Structure, process, and agreement”, en Guest, Greg y MacQueen, Kathleen [eds.], Handbook for team-based qualitative research, Estados Unidos: AltaMira Press.

Ministerio de Desarrollo Social (2017a), Sintesis de resultados. Encuesta de Caracterización Socioeconómica Nacional CASEN - Equidad de Género 2015. Disponible en: http:// observatorio.ministeriodesarrollosocial.gob.cl/casen-multidimensional/casen/docs/ CASEN_2015_Resultados_equidad_genero.pdf [23 de mayo de 2017].

Ministerio de Desarrollo Social (2017b), Sintesis de resultados. Encuesta de Caracterización Socioeconómica Nacional CASEN - Trabajo 2015. Disponible en: http:// observatorio.ministeriodesarrollosocial.gob.cl/casen-multidimensional/casen/docs/ CASEN_2015_Resultados_trabajo.pdf [23 de mayo de 2017].

Ministerio de Salud de Chile (2017), "Protocolo de vigilancia de riesgos psicosociales en el Trabajo”. Disponible en: https://dipol.minsal.cl/departamentos-2/salud-ocupacional/ riesgos-psicosociales/ [23 de marzo de 2018].

Oesch, Daniel et al. (2017), "The Wage Penalty for Motherhood: Evidence on Discrimination from Panel Data and a Survey Experiment for Switzerland”, en Demographic Research, vol. 37, núm. 1, Alemania: Max Planck Society.

OCDE (2018), "Estudios Económicos de la OCDE". Disponible en: https://www. oecd.org/eco/surveys/Chile-2018-OECD-economic-sruvey-Spanish.pdf $[20 \mathrm{de}$ septiembre de 2018].

OIT y PNUD (2009), Trabajo y familia: Hacia nuevas formas de conciliación con corresponsabilidad social, Chile: Organización Internacional del Trabajo.

OIT (2013), Trabajo decente y cuidado compartido. Hacia una propuesta de parentalidad, Chile: OIT/PNUD.

Sabatier, Paul [ed.] (2007), Theories of the policy process, Estados Unidos: Westview Press.

Saldaña, Johnny (2009), The coding manual for qualitative researchers, Inglaterra: Sage.

Schmidt, Vivien (2014), "La política de los pactos sociales y la protección social: ¿importa el discurso?”, en Politicas sociales, núm. 178. Disponible en: http://repositorio.cepal.org/ bitstream/handle/11362/35902/1/S2013402_es.pdf [23 de junio de 2014].

Sisto, Vicente (2009), “Cambios en el trabajo, identidad e inclusión social en Chile: desafíos para la investigación”, en Universum, vol. 24, núm. 2. Disponible en: https://dx.doi. org/10.4067/S0718-23762009000200011 [20 de enero de 2018].

Staab, Silke (2012), "Maternalism, male bias and market reform: historical legacies and current reforms in Chilean social policy”, en Social Politics, vol. 3, núm. 19, Inglaterra: Oxford University Press.

Staab, Silke (2017), Gender and the Politics of Gradual Change: Social Policy Reform and Innovation in Chile, Estados Unidos: Palgrave Macmillan.

Wengraf, Tom (2004), Qualitative Research Interviewing, Inglaterra: Sage. 


\section{Anexo}

\section{Cuadro 1}

\section{Actores entrevistados}

\begin{tabular}{ll}
\hline Actor & Tipo de informante \\
\hline $\begin{array}{l}\text { Organizaciones productivas y y } \\
\text { de servicios (empresas) }\end{array}$ & $\begin{array}{l}\text { Directivos directamente responsables de la gestión de } \\
\text { personas y RR.HH. } \\
\text { Voceros de Asociales Gremiales (ej. Cámaras) del rubro. }\end{array}$ \\
\hline Trabajadores/as & $\begin{array}{l}\text { Sindicatos o grupos negociadores. } \\
\text { Organizaciones de trabajadores por rubro o del nivel } \\
\text { nacional (Central Unitaria de Trabajadores). }\end{array}$ \\
\hline Estado & $\begin{array}{l}\text { Miembros del Poder legislativo: parlamentarios de las } \\
\text { Comisiones de Trabajo de la Cámara y del Senado. } \\
\text { Servidores/as públicos de Ministerios y agencias guberna- } \\
\text { mentales relevantes, como los Ministerios del Trabajo y de } \\
\text { la Mujer y Equidad de Género. }\end{array}$ \\
\hline
\end{tabular}

Fuente: Elaboración propia. 


\section{Cuadro 2}

\section{Macrocategorías de análisis}

\begin{tabular}{ll}
\hline Macrocategoría & Dimensiones conceptuales asociadas \\
\hline Representación de los lími- & Relación trabajo-familia: definición y tipos de medidas \\
tes percibidos entre familia & reconocidas. \\
y trabajo. & Relación trabajo-familia: importancia. \\
& Interacciones negativas familia-trabajo vs. interacciones \\
positivas familia-trabajo.
\end{tabular}

Fuente: Elaboración propia. 
Verónica Gómez-Urrutia. Ph.D. (Gender Studies) por la Universidad de Sussex (Reino Unido). Magíster en Ciencia Política por la Universidad Federal de Minas Gerais (Brasil) y periodista por la Universidad de Chile. Académica de la Facultad de Ciencias Sociales y Humanidades e investigadora asociada al Centro de Estudios y Gestión Social del Maule, ambos pertenecientes a la Universidad Autónoma de Chile, sede Talca, Chile. Líneas de investigación: género y ciudadanía en América Latina, y políticas públicas sobre género y familia. Publicaciones recientes: Gómez-Urrutia, Verónica, Royo, P. y Cruz, M. (2017), "Imagining Families: Gender, Youth, and Diversity in Chile”, en Affilia. Journal of Women and Social Work, vol. 32, núm. 4, Estados Unidos de América, DOI: 10.1177/0886109917718232; Gómez-Urrutia, Verónica, Arellano, O. y Valenzuela, C. (2017), "Negociaciones en familia: género, trabajo y cuidado en Chile", en Revista Estudos Feministas, vol. 25, núm. 2, Brasil, DOI: 10.1590/1806-9584.2017v25n2p661; Jiménez, A., Gómez, Verónica y Palomo, G. (2017), "Work-family balance, participation in family work and parental self-efficacy in Chilean workers", en Canadian Journal of Family and Youth, vol. 9, núm. 1, Canadá.

Andrés Jiménez-Figueroa. Doctor en Administración de Empresas en Universidad Libre de Bruselas, psicólogo organizacional por la Universidad de Santiago de Chile y magíster en Administración y Dirección de Recursos Humanos (Chile). Actualmente se desempeña como académico de la Facultad de Psicología e investigador en el Grupo de Investigación de Calidad de Vida y Ambientes Saludables (GICVAS). Líneas de investigación: conciliación trabajo-familia en organizaciones y calidad de vida en ambientes laborales. Publicaciones recientes: Jiménez, A., Gómez, V. y Palomo, G. (2017), "Work-family balance, participation in family work and parental self-efficacy in Chilean workers", en Canadian Journal of Family and Youth, vol. 9, núm. 1, Canadá; Abarca, S., Letelier, A., Aravena, V. y Jiménez, A. (2016), "Equilibrio trabajo-familia, satisfacción laboral y apoyo familiar en docentes de Escuelas básicas", en Psicología desde el Caribe, vol. 33, núm. 3, Colombia; Jiménez, A. y Aravena Vega, V. (2015), "Desafíos de fomentar estrategias personales e incorporar políticas de conciliación trabajo-familia en las organizaciones”, en Pensamiento Psicológico, vol. 13, núm. 2, Colombia, DOI: 10.11144/Javerianacali.PPSI13-2.dfep 